\title{
PLP1 Gene
}

National Cancer Institute

\section{Source}

National Cancer Institute. PLP1 Gene. NCI Thesaurus. Code C75897.

This gene is involved in neuronal development. 